\title{
Role of alveolar macrophages in asbestosis: modulation of neutrophil migration to the lung after acute asbestos exposure
}

\author{
CARL I SCHOENBERGER, GARY W HUNNINGHAKE, OICHI KAWANAMI, \\ VICTOR J FERRANS, RONALD G CRYSTAL
}

From the Pulmonary Branch and Pathology Branch, National Heart, Lung and Blood Institutes, Bethesda, Maryland, USA

ABSTRACT After intratracheal injection of short chrysotile asbestos fibres in guinea-pigs an intense neutrophil alveolitis was observed within three days. Evaluation by bronchoalveolar lavage of the inflammatory and immune effector cells producing the alveolitis by three days showed an increased proportion of polymorphonuclear leucocytes, which comprised $21 \% \pm 3 \%$ of the total leucocytes compared with $9 \% \pm 2 \%$ for the controls $(p<0.05$ ), persisting for at least six weeks (after which time the polymorphonuclear leucocytes comprised $28 \% \pm 2 \%$ compared with $7 \% \pm 1 \%$ for the controls: $p<0 \cdot 05$ ). One mechanism by which asbestos fibres may cause polymorphonuclear leucocytes to be attracted to the alveolar structures is by induced release of neutrophil chemotactic factor by alveolar macrophages. When exposed in vitro to short or intermediate chrysotile fibres or amosite or crocidolite fibres guinea-pig alveolar macrophages released appreciable amounts of neutrophil chemotactic factor. The release of this chemotactic factor was augmented when the asbestos fibres had been previously exposed to normal serum. The chemotactic factor was lipid soluble, and was similar to the neutrophil chemotactic factor spontaneously released by alveolar macrophages recovered from guinea-pigs exposed in vivo to short chrysotile fibres. These observations suggest that alveolar macrophages may play an important part in the early stages of asbestosis by modulating the migration of neutrophils to the lung.

\begin{abstract}
Asbestosis is a chronic interstitial lung disorder of the alveolar structures characterised by the presence of large numbers of asbestos fibres; an accumulation of inflammatory and immune effector cells ("alveolitis"); alterations in the number, type, and arrangement of parenchymal cells; and interstitial fibrosis. ' Although the aetiology of asbestosis has been known for many years, it is not clear how the asbestos fibre causes the changes in the alveolar structures that characterise the disease. It is now recognised, however, that most non-infectious, nonmalignant diseases of the alveolar structures are mediated through the potent armamentarium of inflammatory and immune effector processes possessed by the cells comprising the alveolitis of these disorders. ${ }^{2}$ In this context, one of the earliest events after inhalation of asbestos fibres is likely to be the accumulation of the effector cells. The present study was designed to characterise the alveolitis of acute asbestosis and to evaluate possible mechanisms through which the asbestos fibres may initiate and maintain the alveolitis.
\end{abstract}

Address for reprint requests: Dr RG Crystal, Building 10, Room 6D06, National Institutes of Health, Bethesda, Maryland 20205, USA.

\section{Methods}

EXPERIMENTAL MODEL

Experimental asbestosis was produced in the NIH strain guinea-pigs (200-300 g) by intratracheal injection of 25 mg of short chrysotile asbestos $(90 \%$ of fibres $<5 \mu \mathrm{m}$ in length; National Institute of Environmental Health Sciences) suspended in $1 \mathrm{ml}$ of sterile $0.9 \%$ saline. The asbestos fibres were autoclaved before suspension. Control animals received either intratracheal saline alone or no injection. Thirty animals were exposed to the asbestos fibres and 40 animals were used as controls. The animals were maintained under standard laboratory conditions in the same room, and were killed three days, two weeks, and six weeks after intratracheal injection.

EVALUATION OF THE ALVEOLITIS

The alveolitis was evaluated by bronchoalveolar lavage and by light microscopy of lung sections.

In half the animals gentle bronchoalveolar lavage was carried out with 5-ml aliquots of sterile saline, to a total of $50 \mathrm{ml}$ per animal. The cells recovered were washed, and 803 
stained cytocentrifuge preparations were made as previously described. ${ }^{3}$ Differential cell counts were performed by counting 200 cells. In the remaining 15 animals the lungs were excised en bloc, inflated to $25 \mathrm{~cm} \mathrm{H}_{2} \mathrm{O}$, fixed with a $10 \%$ buffered formalin solution, and examined morphologically by light microscopy.

EVALUATION OF NEUTROPHIL CHEMOTACTIC FACTOR RELEASE

Normal NIH-strain guinea-pigs weighing 200-300 g were killed, the chest cavities opened, and the lungs perfused with a sterile heparinised saline solution through a cannula inserted into the right ventricle. The lungs were then excised and placed in RPMI-1640 medium for further processing. Teased single cell suspensions were made ${ }^{4}$ and the macrophages isolated by means of HypaqueFicoll density centrifugation. The resulting macrophage suspension consisted of over $95 \%$ alveolar macrophages, of which over $98 \%$ were viable by trypan blue exclusion. The macrophages were then washed twice in Hank's Balanced Salt Solution and then exposed to a solution of $10 \mathrm{mmol} / 1 \mathrm{KHCO}_{3}$ and $0 \cdot 15 \mathrm{~mol} / 1 \mathrm{NH}_{4} \mathrm{Cl}$ to lyse any red cells present. Neutrophil chemotactic factor release by the purified macrophages was evaluated as described by Hunninghake et al. ${ }^{5}$ The macrophages were placed in culture (Costar, 24-well) at a concentration of $10^{6}$ cells $/ \mathrm{ml}$ of RPMI-1640 medium (containing 100 units $/ \mathrm{ml}$ penicillin and $100 \mu \mathrm{g} / \mathrm{ml}$ streptomycin) at $37^{\circ} \mathrm{C}$ for three hours. In addition to the macrophages one of the varieties of asbestos was added to various test wells $-50 \mu \mathrm{g}$ or $100 \mu \mathrm{g}$ of short-range chrysotile ( $90 \%$ of fibres $<5 \mu \mathrm{m}$ long), intermediate-range chrysotile $(90 \%$ of fibres $5-10 \mu \mathrm{m}$ long), amosite, or crocidolite. All fibre samples used in this experiment were standard reference samples obtained from the National Institute of Environmental Health Sciences. Controls consisted of macrophages incubated with washed sepharose particles $(60-140 \mu \mathrm{m}, 50 \mu \mathrm{g} / \mathrm{ml})$ and macrophages incubated with RPMI-1640 medium alone.

After incubation the contents of each culture well were passed through a $0.45 \mu \mathrm{m}$ Millipore filter (Millipore Corporation, Bedford, Massachusetts) to remove all cells and fibres. The resulting supernatants were then assessed for chemotactic activity for neutrophils by means of a modified Boyden chamber and $3 \mu \mathrm{m}$ Nucleopore filters. ${ }^{5}$ Normal human blood neutrophils obtained by dextran sedimentation were used as the responding cells in all studies. Chemotactic factor release was expressed as the number of neutrophils migrating through the chemotaxis membrane per high-power field. All experiments were carried out in duplicate, five oil immersion fields per filter being counted.

To evaluate the effect of serum opsonisation on the ability of the asbestos fibres to stimulate neutrophil chemotactic factor release by macrophages, the four types of asbestos fibres were incubated in parallel for 30 minutes at $37^{\circ} \mathrm{C}$ with normal human serum before being added to the macrophage cultures. The fibre suspensions were then washed extensively in sterile saline to remove any soluble serum components not bound to the asbestos fibres. These serum-coated fibres were then evaluated for their ability to stimulate release of the neutrophil chemotactic factor from alveolar macrophages by the procedure outlined above.

PARTIAL CHARACTERISATION OF THE

CHEMOTACTIC FACTOR

To determine whether the chemotactic activity observed in the supernatants from alveolar macrophages incubated with asbestos fibres was due to the previously described alveolar macrophage-derived chemotactic factor for neutrophils, ${ }^{5-10}$ the extractability of the chemotactic activity into ethyl acetate was evaluated. Extraction was carried out with macrophage supernatants adjusted to $\mathrm{pH}$ $7 \cdot 4$. After extraction the ethyl acetate was dried with a stream of nitrogen and the extracted material resuspended in $5 \mathrm{ml}$ of RPMI-1640. This material was then assayed for chemotactic activity for neutrophils as described above.

CHEMOTACTIC FACTOR RELEASE IN RESPONSE TO IN VIVOEXPOSURE

To evaluate the effect of in vivo exposure to asbestos fibres on the alveolar macrophages, guinea-pigs received intratracheal injections of either $25 \mathrm{mg}$ of short-range chrysotile in $1 \mathrm{ml}$ of saline or $1 \mathrm{ml}$ of saline alone. After three days animals were killed and the macrophages harvested and separated as described above. The macrophages were then evaluated for the spontaneous release of the neutrophil chemotactic factor with the assay described above except that the macrophages from asbestosinjected and saline-injected animals were incubated with medium alone, without further exposure to asbestos in vitro.

STATISTICAL EVALUATION

All data are presented as mean \pm standard error of the mean. All statistical comparisons were made with the two-tailed Student's $t$ test.

\section{Results}

MORPHOLOGY

Guinea-pigs receiving intratracheal injections of asbestos showed appreciable changes in their lungs. Three days after asbestos exposure (fig $1 a$ ) there was a widespread, acute alveolitis, large numbers of neutrophils and 0 eosinophils being found in the alveolar spaces as well as in the interstitial and peribronchial areas. Alveolar macrophages were present in increased numbers. Many alveolar septa showed thickening, probably secondary to interstitial oedema. Some animals showed more focal effects, inflammation being confined to discrete areas within their 


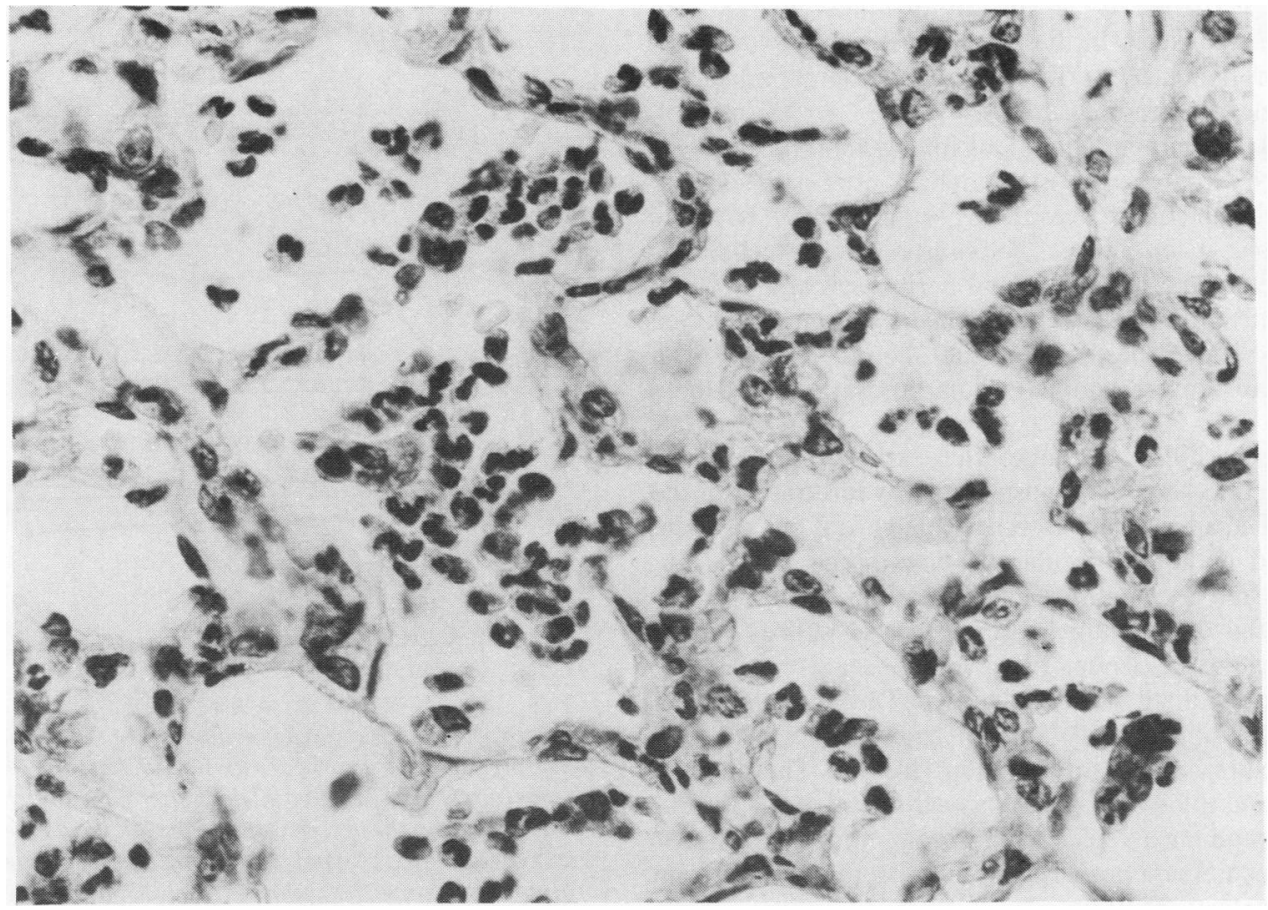

Fig 1 Morphology of the lungs of guinea-pigs after intratracheal exposure to short chrysotile asbestos: (above) from guinea-pig killed three days after intratracheal injection of short chrysotile asbestos, acute alveolitis comprised of monocytes, alveolar macrophages, and polymorphonuclear leucocytes; (below) from control animal that received intratracheal saline, normal lung morphology. (Haematoxylin and eosin, $\times 400$ )

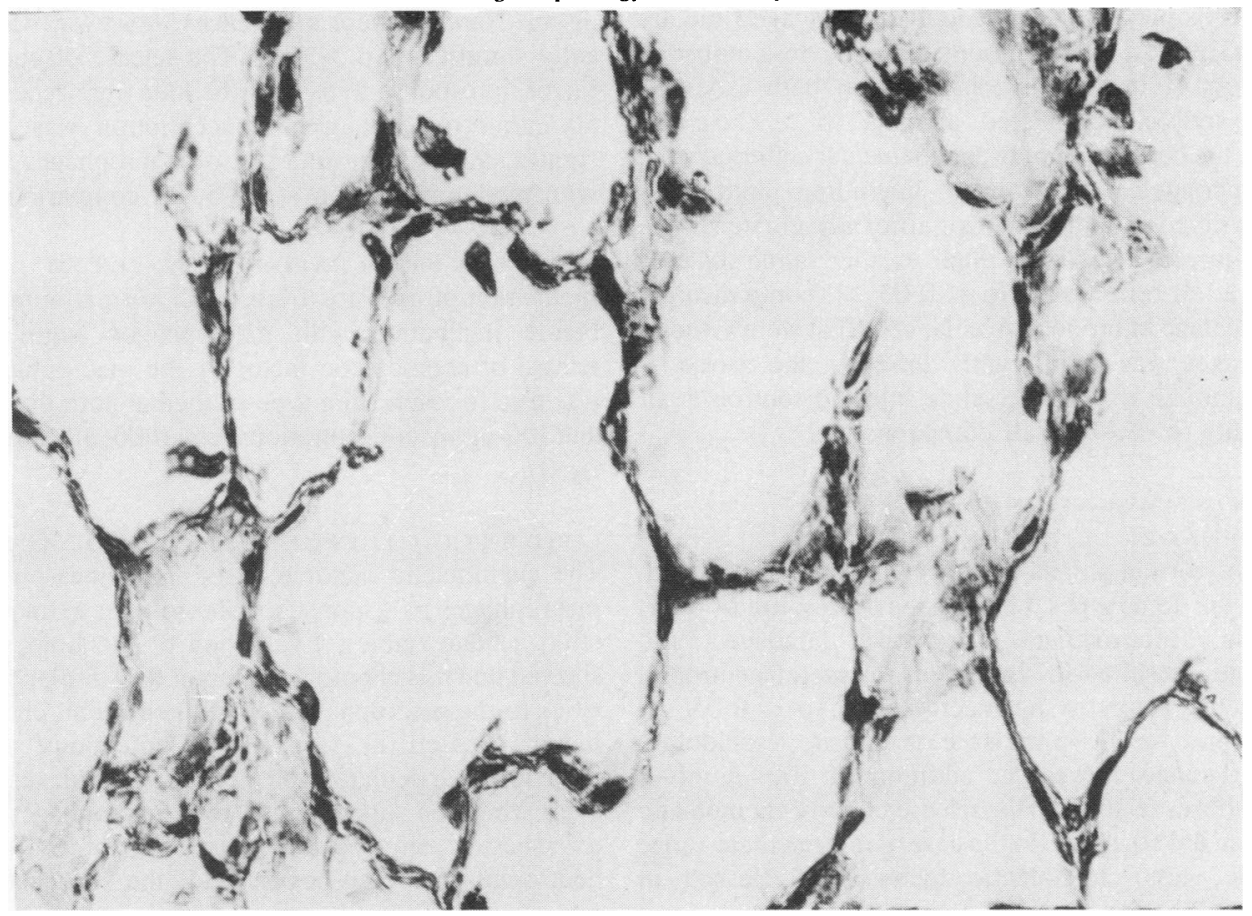


lungs, perhaps as a result of the method of asbestos exposure. In these animals, however, the focal polymorphonuclear infiltration did not predominate in one region rather than others.

At 14 days similar alterations in lung morphology were seen. Neutrophils and eosinophils were still present within the alveolar spaces, along with increased numbers of alveolar macrophages. Polymorphonuclear cells were also seen in the interstitial and peribronchial areas, and the interalveolar septa were thickened. The animals killed 14 days after asbestos exposure showed a more focal pattern of lung inflammation than those killed at three days.

Animals killed 42 days after exposure to asbestos continued to show polymorphonuclear infiltration of the lungs. The areas affected were usually focal but were not confined to any particular portion of the lung. Neutrophils and eosinophils were seen in the alveolar spaces and in the interstitium in subpleural as well as central locations. Thickening of alveolar septa was more pronounced than that seen earlier. The animals which received intratracheal saline showed normal lung architecture and no inflammtion (fig $1 b$ ). The alveolar spaces and interstitial areas showed rare alveolar macrophages, and the alveolar septa were of normal thickness. Peribronchial and perivascular areas were also free from inflammatory infiltrate.

BRONCHOALVEOLAR LAVAGE

The relative proportions of inflammatory and immune effector cells present in bronchoalveolar lavage fluid are shown in fig 2 . At all time points lymphocytes comprised $2 \%$ or less of bronchoalveolar cells in both asbestosinjected and saline-injected animals $(p>0.5)$. By contrast, the percentage of bronchoalveolar cells that were polymorphonuclear leucocytes, including both neutrophils and eosinophils, was significantly greater in the asbestos-injected animals than in the saline-injected controls at all time points ( $p<0.05$, all comparisons). The percentage of bronchoalveolar cells that were alveolar macrophages was significantly lower in the asbestosinjected animals than in the saline-injected controls at all time points ( $p<0.05$, all comparisons).

IN VITRO STIMULATION OF ALVEOLAR

MACROPHAGES

Culture of normal guinea-pig alveolar macrophages with each of the four types of asbestos fibres used-short chrysotile, intermediate chrysotile, amosite, and crocidolite-resulted in significant release of neutrophil chemotactic factor by the macrophages $(p<0.05$, all comparisons: fig 3 ). In the case of amosite, crocidolite, and short-range chrysotile, addition of $100 \mu \mathrm{g} / \mathrm{ml}$ of asbestos fibres resulted in the release of more chemotactic factor than did $50 \mu \mathrm{g} / \mathrm{ml}$. In the case of intermediate-range chrysotile, less chemotactic factor was released in
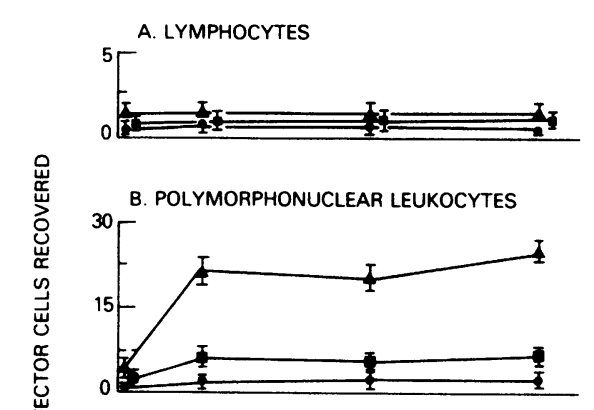

C. ALVEOLAR MACROPHAGES

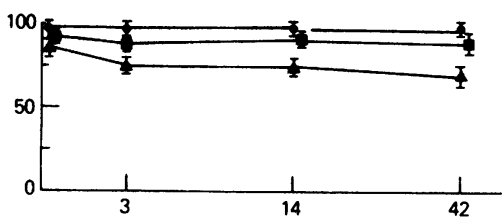

TIME AFTER INJECTION (Days)

Fig 2 Bronchoalveolar lavage evaluation of the alveolitis in guinea-pigs after intratracheal exposure to short chrysotile asbestos fibres, three, 14 , and 42 days after injection: the ordinate indicates the percentage of bronchoalveolar cells that were (a) lymphocytes, (b) polymorphonuclear leucocytes, and (c) alveolar macrophages. Animals received intratracheal asbestos ( $\mathbf{\Delta})$, intratracheal saline ( $\square$ ), or no intratracheal injection (O).

response to $100 \mu \mathrm{g} / \mathrm{ml}$ of asbestos than in response to 50 $\mu \mathrm{g} / \mathrm{ml}$. None of these differences, however, was statistically significant $(p>0 \cdot 1)$. The release of chemotactic factor in response to each of the four fibre types at either $50 \mu \mathrm{g} / \mathrm{ml}$ or $100 \mu \mathrm{g} / \mathrm{ml}$ concentration was, however, significantly greater than that of macrophages incubated with medium alone ( $p<0.05$, all comparions).

EFFECT OF PRIOR EXPOSURE TOSERUM

Incubation of asbestos fibres with normal human serum before incubation with macrophages augmented the release of chemotactic factor by the macrophages. This was true for each fibre type studied at both the $50-\mu \mathrm{g} / \mathrm{ml}$ and $100-\mu \mathrm{g} / \mathrm{ml}$ concentrations $(\mathrm{p}<0.05$, all comparisons: fig 3).

LIPIDEXTRACTION OF CHEMOTACTIC ACTIVITY The chemotactic factor released by guinea-pig alveolar macrophages in response to asbestos was extractable into ethyl acetate (table). Evaluation of the lipid solubility showed that this chemotactic factor had similar characteristics to the macrophage-derived neutrophil chemotactic factor released in response to infectious and noninfectious particulates and immune complexes. ${ }^{6}$ Moreover, while the supernatants from macrophages exposed to asbestos directed the migration of $94.4 \pm 4.8$ neutrophils per high-power field, the supernatants that 


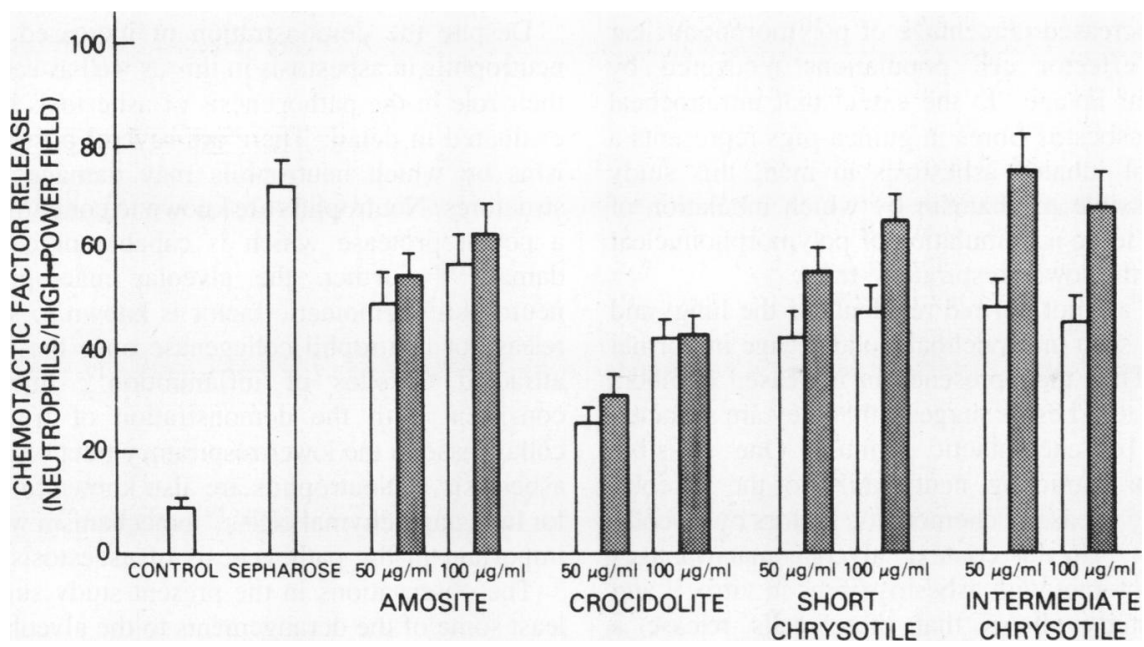

Fig 3 Release of neutrophil chemotactic factor by guinea-pig alveolar macrophages in response to exposure in vitro to asbestos fibres (uncoated, open bars; serum-opsonised, solid bars). "Control" refers to macrophages exposed to medium alone and "sepharose" to macrophages exposed to sepharose beads $(60-140 \mu \mathrm{m}, 50 \mu \mathrm{g} / \mathrm{ml})$, the latter used as a positive control for the release of neutrophil chemotactic factor.

had been extracted with ethyl acetate before testing showed significantly greater chemotactic activity for neutrophils $(p<0.01)$ than for macrophages incubated alone (table).

CHEMOTACTIC FACTOR RELEASEIN VIVO Alveolar macrophages harvested from guinea-pigs that had received intratracheal injections of short chrysotile asbestos three days previously were spontaneously secreting the chemotactic factor for neutrophils (fig 4). In contrast, macrophages from control guinea-pigs injected with saline alone were not spontaneously releasing the chemotactic factor. The difference between the two groups was significant $(p<0.05)$.

\section{Discussion}

Despite the well-documented relationship between inhalation of asbestos fibres and pulmonary fibrosis, the events

Partial characterisation of the neutrophil chemotactic factor released by guinea-pig alveolar macrophages exposed to asbestos fibres*

\begin{tabular}{lc}
\hline Conditions & $\begin{array}{l}\text { Neutrophil chemotactic } \\
\text { factor (neutrophils/ } \\
\text { high-power field }\end{array}$ \\
\hline Macrophages incubated alone & $5 \cdot 7 \pm 2 \cdot 0$ \\
Macrophages incubated with asbestos & $94 \cdot 4 \pm 4 \cdot 8$ \\
Ethyl acetate supernatant & $62 \cdot 8 \pm 5 \cdot 0$ \\
\hline
\end{tabular}

*Short chrysotile ( $100 \mu \mathrm{g} / \mathrm{ml}: 90 \%$ of fibres $<5 \mu \mathrm{m}$ in length)

† Assay performed with a $0 \cdot 3 \mu \mathrm{m}$ chemotaxis membrane with human neutrophils as the responding cells. leading up to this endpoint are still not completely understood. The present study, in an attempt to characterise the early pulmonary events in this disease, has confirmed that acute exposure of the guinea-pig to asbestos fibres results in a polymorphonuclear alveolitis that persists for at least six weeks. During this time there

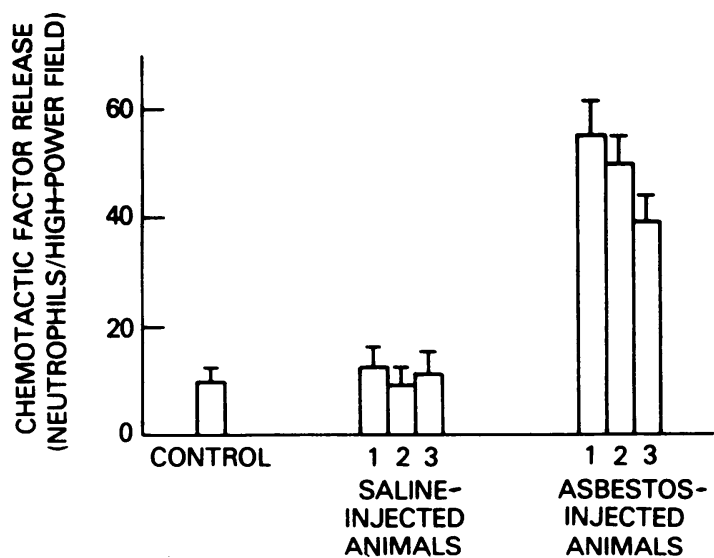

Fig 4 Release of neutrophil chemotactic factor by alveolar macrophages of guinea-pigs that had been given intratracheal injections of short chrysotile fibres three days before (macrophages evaluated in vitro for their spontaneous release of neutrophil chemotactic factor): data for a control animal (no intratracheal injection), three animals given intratracheal asbestos, and three animals given intratracheal saline. The error bars refer to chemotactic factor release for parallel cultures of macrophages from each animal. 
was also an increased percentage of polymorphonuclear cells in the effector cell populations recovered by bronchoalveolar lavage. To the extent that intratracheal instillation of asbestos fibres in guinea-pigs represents a valid model of inhaled asbestosis in man, this study suggests a possible mechanism by which inhalation of asbestos leads to an accumulation of polymorphonuclear leucocytes in the lower respiratory tract.

Neutrophils are not normal residents of the lung, and they are rarely seen in bronchoalveolar lavage in normal individuals. ${ }^{3}$ Thus their presence in increased numbers after exposure to asbestos suggests that they are attracted to the lung by chemotactic stimuli. One possible mechanism for attracting neutrophils to the alveolar structures is the release of chemotactic factors by alveolar macrophages. ${ }^{5-10}$ In this context, alveolar macrophages are known to interact with asbestos fibres in vitro, ${ }^{11}$ and the present study shows that these cells release a neutrophil chemotactic factor in response to four different types of asbestos fibres after in vitro exposure. In addition, alveolar macrophages from guinea-pigs exposed in vivo to short chrysotile fibres spontaneously release the neutrophil chemotactic factor. Furthermore, serum opsonisation significantly enhances release of the neutrophil chemotactic factor by alveolar macrophages. Although it is not clear which serum components are responsible, serum-opsonised asbestos fibres may represent a closer parallel to what happens in vivo since many serum proteins are present within the lower respiratory tract of both normal individuals and patients with interstitial lung disease. ${ }^{12}$

Partial characterisation of the neutrophil chemotactic factor released by guinea-pig alveolar macrophages in response to asbestos fibres showed that the factor was at least partially lipid soluble. This unusual property suggests that it is the same as, or very similar to, the neutrophil chemotactic factor released from both guinea$\mathrm{pig}^{5}$ and human ${ }^{6}$ alveolar macrophages already described. A similar factor has also been implicated in the pathogenesis of idiopathic pulmonary fibrosis, another chronic interstitial lung disease in which neutrophil accumulation appears to play an important part. ${ }^{13}$

In addition to the chemotactic factor derived from alveolar macrophages, another possible mechanism by which asbestos fibres might attract neutrophils to the alveolar structures is the generation of complementderived chemotactic factors. Previous studies have shown that asbestos fibres can activate complement by both the classical $^{14}$ and the alternative ${ }^{15}$ pathways in vitro, and further that this is followed by the generation of complement-derived chemotactic factors for "peripheral blood leucocytes." Since complement components are known to be normally present in lower respiratory tract secretions, ${ }^{12}$ these proteins are likely to interact with asbestos fibres in vivo, and may generate chemotactic factors for polymorphonuclear leucocytes.
Despite the demonstration of increased numbers of neutrophils in asbestosis in this as well as earlier studies, their role in the pathogenesis of asbestosis has not been evaluated in detail. There are several possible mechanisms by which neutrophils may damage the alveolar structures. Neutrophils are known to contain collagenase, a potent protease which is capable of causing tissue damage. ${ }^{16}$ Further, the alveolar macrophage-derived neutrophil chemotactic factor is known to stimulate the release of neutrophil collagenase once the neutrophil is attracted to sites of inflammtion ${ }^{17}$; this finding is consistent with the demonstration of free neutrophil collagenase in the lower respiratory tract of patients with asbestosis. ${ }^{18}$ Neutrophils are also known to be cytotoxic for lung parenchymal cells, ${ }^{19}$ a mechanism which may be important in the pathogenesis of asbestosis.

The observations in the present study suggest that at least some of the derangements to the alveolar structures associated with asbestosis are mediated by the ability of the asbestos fibre to stimulate alveolar macrophages to attract neutrophils to the lower respiratory tract.

\section{References}

' Selikoff IJ, Hammond EC. Health hazards of asbestos exposure. Ann NY Acad Sci 1979;330:1-814.

${ }^{2}$ Crystal RG, Gadek JE, Ferrans VJ, Fulmer JD, Line BR, Hunninghake $\mathrm{GW}$. Interstitial lung disease: current concepts of pathogenesis, staging and therapy. Am J Med 1981;70:542-68.

${ }^{3}$ Hunninghake GW, Gadek JE, Kawanami O, Ferrans VJ, Crystal RG. Inflammatory and immune processes in the human lung in health and disease: evaluation by bronchoalveolar lavage. Am J Pathol 1979;97:149-206.

${ }^{4}$ Hunninghake GW, Fauci AS. Immunologic reactivity of the lung. I. A guinea-pig model for the study of pulmonary mononuclear cell subpopulations. Cell Immunol 1976; 26:89-97.

5 Hunninghake GW, Gallin JI, Fauci AS. Immunologic reactivity of the lung: the in vivo and in vitro generation of a neutrophil chemotactic factor by alveolar macrophages. Am Rev Respir Dis 1978;117:15-23.

${ }^{6}$ Hunninghake GW, Gadek JE, Fales HM, Crystal RG. Human alveolar macrophage-derived chemotactic factor for neutrophils: stimuli and partial characterization. J Clin Invest 1980;66:473-83.

${ }^{7}$ Kazmierowski JA, Gallin JI, Reynolds HY. Mechanism for the inflammatory response in primate lungs: demonstration and partial characterisation of an alveolar macrophagederived chemotactic factor with preferential activity for polymorphonuclear leucocytes. J Clin Invest 1977;59:27381.

${ }^{8}$ Gadek JE, Hunninghake GW, Zimmerman RL, Crystal RG. Regulation of the release of alveolar macrophage-derived neutrophil chemotactic factor. Am Rev Respir Dis 1980;121:723-33.

${ }^{9}$ Merrill WW, Nagel GP, Matthay RA, Reynolds HY. Alveolar macrophage-derived chemotactic factor: kinetics of the in 
vitro production and partial characterisation. J Clin Invest 1980;65:268-76.

${ }^{10}$ Lugano EM, Dauber JH, Daniele RP. Silica stimulation of chemotactic factor release by alveolar macrophages. Am Rev Respir Dis 1980;121:81.

1 Miller K. The effects of asbestos on macrophages. CRC Crit Rev Toxicol 1978;5:319-54.

${ }^{12}$ Bell DY, Haseman JA, Spock A, McLennan G, Hook GER. Plasma proteins of the bronchoalveolar surface of the lungs of smokers and nonsmokers. Am Rev Respir Dis 1981;124:72-9.

${ }^{13}$ Hunninghake GW, Gadek JE, Lawley TJ, Crystal RG. Mechanisms of neutrophil accumulation in the lungs of patients with idiopathic pulmonary fibrosis. J Clin Invest 1981;68:259-69.

${ }^{14}$ Hasselbacher P. Binding of immunoglobulin and activation of complement by asbestos fibers. J Allergy Clin Immunol 1979;64:294-8.

${ }^{15}$ Wilson MR, Gaumer HR, Salvaggio JE. Activation of the alternative complement pathway and generation of chemotactic factors by asbestos. J Allergy Clin Immunol 1977;60:218-22.

${ }^{16}$ Krane SM. Degradation of collagen in connective tissue diseases. Rheumatoid arthritis. In: Burleigh RMC, Poole AR, eds. Dynamics of connective tissue macromolecules. Amsterdam: Elsevier-North Holland Publishing Company, 1975:309-24.

${ }^{17}$ Gadek JE, Fells G, Hunninghake GW, Crystal RG. Interaction of the alveolar macrophage (AM) and the circulating neutrophil: AM-induced neutrophil activation. Am Rev Respir Dis 1979;119:66 (abstract).

${ }^{18}$ Gadek J, Hunninghake G, Schoenberger C, Fells G, Crystal R. Pulmonary asbestos and idiopathic pulmonary fibrosis: pathogenetic parallels. Chest 1981;80, suppl:635.

${ }^{19}$ Martin WJ II, Gadek JE, Hunninghake GW, Crystal RG. Polymorphonuclear leucocyte-induced lung injury: a quantitative in vitro model. Clin Res 1981;29:449 (abstract). 